\title{
Sums of Darboux and continuous functions
}

\author{
by
}

Juris Steprāns (Toronto, Ont.)

\begin{abstract}
It is shown that for every Darboux function $F$ there is a non-constant continuous function $f$ such that $F+f$ is still Darboux. It is shown to be consistent- the model used is iterated Sacks forcing - that for every Darboux function $F$ there is a nowhere constant continuous function $f$ such that $F+f$ is still Darboux. This answers questions raised in [5] where it is shown that in various models of set theory there are universally bad Darboux functions, Darboux functions whose sum with any nowhere constant, continuous function fails to be Darboux.
\end{abstract}

1. Introduction. A function which maps any connected set to a connected set is known as a Darboux function. This paper will be concerned with functions from $\mathbb{R}$ to $\mathbb{R}$ and, in this context, Darboux simply means that the image of any interval is an interval. While there are various results establishing similarities between continuous functions and Darboux functions of first Baire class, the fact that it is possible to construct Darboux functions by transfinite induction allows all sorts of pathologies to exist. For example, transfinite induction can be used to construct a Darboux function $F$ such that the function $F(x)+x$ is not Darboux [8]. In [6] it is shown that if $\mathcal{G}$ is a family of functions such that card $|\mathcal{G}|^{+}<2^{\aleph_{0}}$ then there is a Darboux function $F$ such that $F+g$ is not Darboux for all $g \in \mathcal{G}$. This result is extended in [5] where it is established, assuming certain set theoretic hypotheses, that there exists a universally bad Darboux function $f: \mathbb{R} \rightarrow \mathbb{R}$, which means that, for every nowhere constant continuous $g: \mathbb{R} \rightarrow \mathbb{R}, f+g$ does not have the Darboux property. In unpublished work W. Weiss has shown that a universally bad Darboux function can be constructed assuming only the existence of a $2^{\aleph_{0}}$ additive ideal $\mathcal{I}$ on $\mathcal{B}$, the Borel subsets of $\mathbb{R}$, such that the Boolean algebra $\mathcal{B} / \mathcal{I}$ has the $2^{\aleph_{0}}$ chain condition; in other words, there do not exist $2^{\aleph_{0}}$ elements of $\mathcal{B}$ whose pairwise intersections belong to $\mathcal{I}$.

In this paper it will be shown that some form of set theoretic hypothesis

1991 Mathematics Subject Classification: 26A15, 03C55.

This research was partially supported by NSERC. 
used is necessary for such a result because there is a model of set theory where for every Darboux function $F$ there is a nowhere constant continuous function $f$ such that $F+f$ is also Darboux. The significance of the adjective "nowhere constant" in this statement requires some comment because it might seem a minor point. An indication that this is not so is given by the fact that, in spite of having shown that there is a Darboux function $f$ such that for every nowhere constant continuous $g: \mathbb{R} \rightarrow \mathbb{R}, f+g$ does have the Darboux property, the authors of [5] pose the following question at the end of their paper.

Question 1.1. Does there exist a Darboux function $F: \mathbb{R} \rightarrow \mathbb{R}$ such that $F+g$ does not have the Darboux property whenever $g$ is continuous but not constant?

Section 2 provides a negative answer to this problem. Section 3 contains some technical material on Sacks forcing and Section 4 makes use of this material in proving the main consistency result. The final section contains some open questions.

2. Sums with non-constant functions. The next lemma follows directly from Theorem 1.1 of [2] - a proof is presented here only for the convenience of the reader.

Lemma 2.1. If $F$ is Darboux and not continuous at $x$ then there is an interval $(a, b) \neq \emptyset$ such that for each $y \in(a, b)$ there is a sequence $\left\{x_{n} \mid\right.$ $n \in \omega\}$ such that $\lim _{n \rightarrow \infty} x_{n}=x$ and $F\left(x_{n}\right)=y$ for all $n \in \omega$.

Proof. Because $F$ is not continuous at $x$ there are sequences $\left\{y_{n}^{a}\right\}_{n \in \omega}$ and $\left\{y_{n}^{b}\right\}_{n \in \omega}$ such that

$$
\lim _{n \rightarrow \infty} y_{n}^{a}=x=\lim _{n \rightarrow \infty} y_{n}^{b} \quad \text { and } \quad \lim _{n \rightarrow \infty} F\left(y_{n}^{a}\right)=a<b=\lim _{n \rightarrow \infty} F\left(y_{n}^{b}\right) .
$$

Given $n \in \omega$ and $y \in(a, b)$ let $k$ be such that $\left|y_{k}^{a}-x\right|<1 / n,\left|y_{k}^{b}-x\right|<1 / n$ and $F\left(y_{k}^{b}\right)>y>F\left(y_{k}^{a}\right)$. Then use the Darboux property of $F$ to find $x_{n}$ between $y_{k}^{a}$ and $y_{k}^{b}$ such that $F\left(x_{n}\right)=y$.

COROLlaRY 2.1. If $F$ is a Darboux function which is finite-to-one then $F$ is continuous.

Lemma 2.2. If $F: \mathbb{R} \rightarrow \mathbb{R}$ is a Darboux function which is continuous at only countably many points then there is a non-constant, continuous function $f$ such that $F+f$ is Darboux.

Proof. To each real $x$ at which $F$ is not continuous, use Lemma 2.1 to assign an interval $\left(a_{x}, b_{x}\right)$ such that for each $y \in\left(a_{x}, b_{x}\right)$ there is a sequence $\left\{x_{n} \mid n \in \omega\right\}$ such that $\lim _{n \rightarrow \infty} x_{n}=x$ and $F\left(x_{n}\right)=y$ for all $n$. For rationals $p$ and $q$ let $X(p, q)$ be the set of all $x$ such that $a_{x}<p<q<b_{x}$ and note 
that $X(p, q)$ is a closed set. Because $F$ is continuous at only countably many points, it cannot be the case that $X(p, q)$ is nowhere dense for each pair of rationals $p$ and $q$. Therefore let $[s, t]$ and $[a, b]$ be intervals such that $\left[a_{x}, b_{x}\right] \supseteq[a, b]$ for each $x \in[s, t]$ and, furthermore, $F(s)=a$ and $F(t)=b$. Observe that $F^{-1}\{y\}$ is dense in $[s, t]$ for each $y \in[a, b]$.

Next, choose a family of open intervals $\mathcal{I}$ such that

- $\mathcal{I}=\bigcup_{n \in \omega} \mathcal{I}_{n}$ where $\mathcal{I}_{n}=\left\{\left(u_{i}^{n}, v_{i}^{n}\right) \mid i \in 2^{n}-1\right\}$,

- $v_{i}^{n}<u_{i+1}^{n}$ for all $n$ and $i \in 2^{n}-1$,

- $u_{i}^{n}=u_{2 i+1}^{n+1}$ and $v_{i}^{n}=v_{2 i+1}^{n+1}$ for each $n$ and $i \in 2^{n}-1$,

- $\bigcup \mathcal{I}$ is dense in $[s, t]$,

- $F\left(u_{i}^{n}\right)=a$ and $F\left(v_{i}^{n}\right)=b$ for each $n$ and $i$,

- $\sup F \uparrow\left[v_{i-1}^{n}, u_{2 i}^{n+1}\right] \cup\left[v_{2 i}^{n+1}, u_{i}^{n}\right] \leq \sup F\left\lceil\left[u_{2 i}^{n+1}, v_{2 i}^{n+1}\right]+1 /(n+1)\right.$ where it is understood that, in the case $i=0, v_{-1}^{n}=s$ and, in the case $i=2^{n}-1$, $u_{2^{n}-1}^{n}=t$,

- $\inf F \uparrow\left[v_{i-1}^{n}, u_{2 i}^{n+1}\right] \cup\left[v_{i}^{n+1}, u_{2 i}^{n}\right] \geq \inf F \uparrow\left[u_{2 i}^{n+1}, v_{2 i}^{n+1}\right]-1 /(n+1)$, again with $v_{-1}^{n}=s$ and $u_{2^{n}-1}^{n}=t$.

Now let $g$ be a non-decreasing, continuous function such that $g\lceil I$ is constant for each $I \in \mathcal{I}, g(s)=0, g(t)=b-a$ and $g$ is constant on $(-\infty, s]$ and $[t, \infty)$. The reader who insists on concreteness may verify that

$$
g(x)=\sup \left\{i / 2^{n} \mid u_{i}^{n} \leq x\right\}(b-a)
$$

satisfies these requirements.

To see that $F+g$ is Darboux suppose that $x<y$ and $w$ lies between $F(x)+g(x)$ and $F(y)+g(y)$. First of all observe that it may be assumed that $s \leq x<y \leq t$. The reason it may be assumed that $s \leq x$ is that if $x<s$ then either $w$ lies between $F(x)+g(x)$ and $F(s)+g(s)$ or else it lies between $F(s)+g(s)$ and $F(y)+g(y)$. In order to eliminate the first case use the fact that $F$ is Darboux and $g$ is constant on $[x, s]$ to find $z \in[x, s]$ such that $F(z)+g(z)=w$. In the second case it may, of course, be assumed that $x=s$. A similar argument can be applied to show that, without loss of generality, $y \leq t$.

First consider the case where there is some $(u, v) \in \mathcal{I}$ such that $g$ has constant value $c$ on $(u, v)$ and $w \in(a+c, b+c)$ and such that $x \leq u<v \leq y$. Use the fact that $g$ is constant on $[u, v], F$ is Darboux, $F(u)=a$ and $F(v)=b$ to find $z \in(u, v)$ such that $F(z)+g(z)=w$.

In the remaining case it follows from the fact that $\bigcup \mathcal{I}$ is dense and the continuity of $g$ that either $w \geq b+g(r)$ for every $r \in \bigcup \mathcal{I} \cap(x, y)$ or $w \leq a+g(r)$ for every $r \in \bigcup \mathcal{I} \cap(x, y)$. Only the first case will be considered since the other one is dealt with similarly. Furthermore, it will be assumed that $F(x)+g(x)<F(y)+g(y)$ since the other case is also similar. To begin, suppose that $y \in(u, v) \in \mathcal{I}$. Since $F(u)=a<b \leq w-g(y)$ it follows that 
$F(u)<w-g(y)<F(y)$ and so it is possible to appeal to the Darboux property of $F$ and the constancy of $g$ on $[u, v]$.

On the other hand, if $y \notin \bigcup \mathcal{I}$ then there must be some $m \in \omega$ such that $F(y)+g(y)-w<1 / m$. Choose $\delta>0$ such that $y-x>\delta$ and $g(y)-g(r)>$ $1 /(2 m)$ if $y-r<\delta$. Then there is some $k \geq 2 m$ such that $\left(u_{i}^{k}, v_{i}^{k}\right) \in \mathcal{I}_{k}$ and $y-\delta \leq u_{i}^{k}<v_{i}^{k}<y \leq u_{i / 2}^{k-1}$ for some $i \in 2^{k}-1$. It follows that $F(y) \leq \sup F\lceil$ $\left[v_{i}^{k}, u_{i}^{k-1}\right]<\sup F \uparrow\left[u_{i}^{k}, v_{i}^{k}\right]+1 / k$ and hence $\sup \left\{F(r)+g(r) \mid r \in\left[u_{i}^{k}, v_{i}^{k}\right]\right\}>$ $F(y)+g(y)-1 / m$. Therefore $w<\sup \left\{F(r)+g(r) \mid r \in\left[u_{i}^{k}, v_{i}^{k}\right]\right\}$. In the case being considered, it follows that $w \geq b+g\left(u_{i}^{k}\right)>a+g\left(u_{i}^{k}\right)=F\left(u_{i}^{k}\right)+g\left(u_{i}^{k}\right)$; in other words, there is some $r \in\left[u_{i}^{k}, v_{i}^{k}\right]$ such that

$$
F\left(u_{i}^{k}\right)+g\left(u_{i}^{k}\right)<w<F(r)+g(r) .
$$

Because $g$ is constant on $\left[u_{i}^{k}, v_{i}^{k}\right]$ it now follows from the Darboux property of $F$ that there is $z \in\left[u_{i}^{k}, v_{i}^{k}\right]$ such that $F(z)+g(z)=w$.

LEMMA 2.3. If $F$ is a Darboux function which is continuous on an uncountable set then there is a continuous, non-constant function $g$ such that $F+g$ is Darboux.

Proof. Because the set of points where $F$ is continuous is Borel, it is possible to find a perfect, nowhere dense set $P$ such that $F$ is continuous at each point of $P$. Then $\mathbb{R} \backslash P=\bigcup \mathcal{I}$ where $\mathcal{I}$ is a disjoint family of open intervals of order type the rationals. Let $g$ be any continuous, non-decreasing function which is not constant, yet $g$ has constant value $g_{I}$ on each interval $I \in \mathcal{I}$.

To see that $F+g$ is Darboux suppose that $x<y$ and that $F(x)+g(x)<$ $w<F(y)+g(y)$; a similar proof works if $F(x)+g(x)>w>F(y)+g(y)$. If there is some interval $I \in \mathcal{I}$ such that $I \subseteq[x, y]$ and $\sup F\left\lceil I \geq w-g_{I} \geq\right.$ $\inf F\lceil I$ then the Darboux property of $F$ guarantees that there is some $z \in I$ such that $F(z)+g(z)=w$.

If there is no such $I$ then consider first the case where there are $I$ and $J$ in $\mathcal{I}$ such that $I \subseteq[x, y], J \subseteq[x, y], \sup F\left\lceil I<w-g_{I}\right.$ and $\inf F\left\lceil J>w-g_{J}\right.$ and suppose that $\sup I<\inf J$. Let $z$ be the infimum of all intervals $J^{\prime}$ such that $\sup I<\inf J^{\prime}$ and $w-g_{J^{\prime}}<\inf F\left\lceil J^{\prime}\right.$. First observe that $z \notin \bigcup \mathcal{I}$ and so $F$ is continuous at $z$ and hence

$$
w-g(z) \leq \liminf _{r \rightarrow z^{+}} F(r)=\lim _{r \rightarrow z^{+}} F(r)=F(z) .
$$

Notice that this immediately implies $z$ is not the right-hand end point of any interval in $\mathcal{I}$ because if $N=(\eta, z) \in \mathcal{I}$ then $g_{N}=g(z)$ and so

$$
\sup F\left\lceil N<w-g_{N}=w-g(z) \leq F(z)\right. \text {, }
$$

contradicting the continuity of $F$ at $z$. Now, since $g$ is also continuous at $z$ and there is a sequence of intervals from $\mathcal{I}$ converging to $z$, it follows from 
the defining property of $z$ that

$$
w-g(z) \geq \liminf _{r \rightarrow z^{-}} F(r)=\lim _{r \rightarrow z^{-}} F(r)=F(z)
$$

and so $F(z)+g(z)=w$. Similar arguments in the other cases establish that one of the following two possibilities holds:

- if $I \subseteq[x, y]$ then $\sup F\left\lceil I<w-g_{I}\right.$,

- if $I \subseteq[x, y]$ then $\inf F\left\lceil I>w-g_{I}\right.$.

Consider the first alternative. If $y \notin \bigcup \mathcal{I}$ then $F$ is continuous at $y$ and so

$$
\lim _{s \rightarrow y^{-}}(F(s)+g(s))=F(y)+g(y) \leq w
$$

and this is impossible because $F(y)+g(y)>w$. If $y \in(a, b) \in \mathcal{I}$ and $F(a)+g(a)>w$ then the same argument applies because $a \notin \bigcup \mathcal{I}$. On the other hand, if $F(a)+g(a) \leq w$ then the Darboux property of $F$ and the fact that $g$ is constant on $[a, b]$ yield $z \in[a, y)$ such that $F(z)+g(z)=w$. The other alternative is dealt with similarly.

TheOREM 2.1. If $F$ is a Darboux function then there is a non-constant continuous function $g$ such that $F+g$ is Darboux.

Proof. Either $F$ is continuous on an uncountable set or it is not. If it is, use Lemma 2.3 and if it is not then use Lemma 2.2.

3. Sacks reals. The Sacks partial order of perfect trees will be denoted by $\mathbb{S}$ and the iteration, of length $\xi$, of this partial order will be denoted by $\mathbb{S}_{\xi} ;$ so $\mathbb{S}_{1}=\mathbb{S}$ and $\mathbb{S}_{0}=\emptyset$. For other notation and definitions concerning Sacks reals see [7] as well as [1]. For any $p \in \mathbb{S}_{\xi}$ define

$p^{*}=\left\{\theta: \xi \times \omega \rightarrow 2 \mid\left(\forall F \in[\xi]^{<\aleph_{0}}\right)(\forall m \in \omega)(\theta\lceil F \times m\right.$ is consistent with $p)\}$. It is easy to see $p^{*} \subseteq 2^{\xi \times \omega}$ is a closed set; but there is no reason to believe that it should be non-empty. However, if $p$ is determined (see p. 580 of [7] for a definition) then $p^{*}$ is a reasonably accurate reflection of $p$. In [7] a notion very similar to $p^{*}$ is defined and denoted by $E_{p}$. The only difference is that $E_{p} \subseteq\left(2^{\omega}\right)^{A}$ where $A$ is the domain of $p$. The projection function from $2^{\xi \times \omega}$ to $2^{\gamma \times \omega}$ will be denoted by $\Pi_{\xi, \gamma}$.

Lemma 3.1. If $p \in \mathbb{S}_{\xi}$ is $(E, k)$-determined and $p \Vdash_{\mathbb{S}_{\alpha}}$ " $x \in \mathbb{R} \backslash V$ " then for each $E \in[\alpha]^{<\aleph_{0}}$ and $k \in \omega$ there is $q$ such that $(q, k) \leq_{E}(p, k)$ and a function $Z: q^{*} \rightarrow \mathbb{R}$ such that

(1) $q \Vdash " x=Z(G) "$,

(2) $Z(x) \neq Z(y)$ unless $\Pi_{\omega_{2}, 1}(x)=\Pi_{\omega_{2}, 1}(y)$.

Proof. This is essentially Lemma 6 on p. 580 of [7]. The only difference is that it is now required that $(q, k) \leq_{E}(p, k)$ whereas Miller's Lemma 6 only asserts that $q \leq p$. On the other hand, the assertion required here is only that 
$Z(x) \neq Z(y)$ unless $\Pi_{\omega_{2}, 1}(x)=\Pi_{\omega_{2}, 1}(y)$; whereas a canonical condition for $x$, in Miller's terminology, actually yields a one-to-one function $Z$. The way around this is to choose for each $\sigma: E \times k \rightarrow 2$ a condition $q_{\sigma}$ and a one-toone function $Z_{\sigma}:\left(q_{\sigma}\lceil\beta(\sigma))^{*} \rightarrow \mathbb{R}\right.$ such that $q_{\sigma} \Vdash " x=Z_{\sigma}\left(\Pi_{\omega_{2}, \beta(\sigma)}(G)\right)$ ". The point to notice is that the domain of $Z_{\sigma}$ depends on $\beta(\sigma)$ and so there may not be a single ordinal which works for all $\sigma$. Nevertheless, $\beta(\sigma) \geq 1$ for each $\sigma$ and so it is possible to define $Z=\bigcup_{\sigma} Z_{\sigma} \circ \Pi_{\omega_{2}, \beta(\sigma)}$. It follows that $Z(x) \neq Z(y)$ unless $\Pi_{\omega_{2}, 1}(x)=\Pi_{\omega_{2}, 1}(y)$.

Lemma 3.2. If $p \in \mathbb{S}_{\xi}$ is $(E, k)$-determined and $F: p^{*} \rightarrow \mathbb{R}$ and $G: p^{*} \rightarrow$ $\mathbb{R}$ are continuous functions such that $F\left\lceil q^{*} \neq G\left\lceil q^{*}\right.\right.$ for each $q \leq p$ then there is some $q$ such that $(q, k)<_{E}(p, k)$ and the images of $q^{*}$ under $F$ and $G$ are disjoint.

Proof. Let $\Sigma$ be the set of all $\sigma: E \times k \rightarrow 2$ which are consistent with $p$. For each $\sigma \in \Sigma, F\left\lceil(p \mid \sigma)^{*} \neq G\left\lceil p \mid \sigma^{*}\right.\right.$ and so it is possible to find some $x_{\sigma} \in(p \mid \sigma)^{*}, E_{\sigma} \in[\xi]^{<\aleph_{0}}$ and $k_{\sigma} \geq k$ such that $F\left(x_{\sigma}\right) \neq G\left(x_{\sigma}\right)$ and, moreover, the image of $\left(\left(p \mid x_{\sigma}\left\lceil E_{\sigma}\right) \times k_{\sigma}\right)^{*}\right.$ under $F$ is disjoint from the image under $G$. Let $q^{\prime}=\bigcup_{\sigma \in \Sigma}\left(\left(p \mid x_{\sigma}\left\lceil E_{\sigma}\right)_{\sigma} \times k_{\sigma}\right)\right.$. By repeating this operation for each pair $\{\sigma, \tau\} \in[\Sigma]^{2}$ it is possible to obtain $q$ with the desired properties.

\section{Darboux functions and the Sacks model}

Lemma 4.1. If $H: I \rightarrow \mathbb{R}$ is Darboux then there is a countable set $D$ such that, for any continuous function $F$, if for every $a \in D$ and $b \in D$ and $t$ such that

$$
H(a)+F(a)<t<H(b)+F(b)
$$

there is some $c$ between $a$ and $b$ such that $H(c)+F(c)=t$ then $H+F$ is also Darboux.

Proof. Let $D$ be any countable set such that $H\lceil D$ is dense in the graph of $H$ and suppose that $F$ is continuous. If $F(x)+H(x)<t<F(y)+H(y)$ then, because $F$ is continuous at both $x$ and $y, H$ is Darboux and $H \uparrow D$ is dense in the graph of $H$ there are $d_{x} \in D$ and $d_{y} \in D$, between $x$ and $y$, such that $F\left(d_{x}\right)+H\left(d_{x}\right)<t<F\left(d_{y}\right)+H\left(d_{y}\right)$. Hence, if there is some $z$ between $d_{x}$ and $d_{y}$ such that $H(z)+F(z)=t$ then $z$ also lies between $x$ and $y$.

For the rest of this section by a condition in $\mathbb{S}_{\xi}$ will be meant a determined condition. Real-valued functions will be considered to have as their domain the unit interval $I$. This is merely a convenience that allows the use of the complete metric space of all continuous real-valued functions on the unit interval using the sup metric. This space will be denoted by $\mathcal{C}(I, \mathbb{R})$ and its metric will be $\varrho(f, g)=\sup \{|f(x)-g(x)|: x \in I\}$. 
THEOREM 4.1. Let $V$ be a model of $2^{\aleph_{0}}=\aleph_{1}$ and $V[G]$ be obtained by adding $\omega_{2}$ Sacks reals with countable support iteration. If $H: I \rightarrow \mathbb{R}$ is a Darboux function in $V[G]$ then there is a second category set of continuous functions $f$ such that $H+f$ is also Darboux.

Pr o of. If the theorem fails then, in $V[G]$, let $H$ be a Darboux function and $X$ be a comeagre subset of $\mathcal{C}(I, \mathbb{R})$ which provide a counterexample. Let $D$ be a countable set, provided by Lemma 4.1 , such that for any continuous function $F$, if for every $a \in D$ and $b \in D$ and $t$ such that $H(a)+F(a)<t<$ $H(b)+F(b)$ there is some $c$ between $a$ and $b$ such that $H(c)+F(c)=t$ then $H+F$ is also Darboux. It must be true that, for each continuous function $g \in X$ there is an interval $N(g)=[a, b]$, with endpoints in $D$, and a real $T(g)$ between $H(a)+g(a)$ and $H(b)+g(b)$ such that there is no $z \in[a, b]$ such that $H(z)+g(z)=T(g)$.

By a closure argument, there must exist $\alpha \in \omega_{2}$ such that

- $D \in V\left[G \cap \mathbb{S}_{\alpha}\right]$,

- $T(f) \in V\left[G \cap \mathbb{S}_{\alpha}\right]$ for every $f \in X$ which belongs to $V\left[G \cap \mathbb{S}_{\alpha}\right]$,

- if $x$ is in $V\left[G \cap \mathbb{S}_{\alpha}\right]$ then so is $H(x)$,

- $X=\bigcap_{n \in \omega} U_{n}$ where each $U_{n}$ is a dense open set belonging to $V\left[G \cap \mathbb{S}_{\alpha}\right]$.

To simplify notation it may be assumed that $V=V\left[G \cap \mathbb{S}_{\alpha}\right]$. In $V$, let $\left\{d_{i} \mid i \in \omega\right\}$ enumerate $D$, let $G$ denote the generic function from $\omega_{2} \times \omega$ to 2 which is obtained from an $\mathbb{S}_{\omega_{2}}$ generic set, and let $p_{0} \in \mathbb{S}_{\omega_{2}}$ be a determined condition.

Let $\mathfrak{M}$ be a countable elementary submodel of $\left(H\left(\omega_{3}\right), \in\right)$ containing the functions $T$ and $N$ and the name $H$. Let $\left\{E_{n} \mid n \in \omega\right\}$ be an increasing sequence of finite sets such that $\bigcup_{n \in \omega} E_{n}=\mathfrak{M} \cap \omega_{2}$. (The use of the elementary submodel is only a convenience that allows the finite set $E_{n}$ to be chosen before beginning the fusion argument, thereby avoiding some bookkeeping.) Construct, by induction on $n \in \omega$, functions $f_{n}$, as well as conditions $p_{n} \in \mathbb{S}_{\omega_{2}}$, reals $\varepsilon_{n}>0$ and integers $k_{n}$, all in $\mathfrak{M}$, such that:

$\operatorname{IH}(0) \quad f_{n} \in \mathcal{C}(I, \mathbb{R})$ and $f_{0}$ is chosen arbitrarily,

$\mathrm{IH}(1) \quad$ the neighbourhood of $f_{n}$ of radius $\varepsilon_{n}<1 / n$ in $\mathcal{C}(I, \mathbb{R})$ is contained in $\bigcap_{i \leq n} U_{i}$

$\operatorname{IH}(2) \quad \varrho\left(f_{n}, f_{n+1}\right)<\varepsilon_{n} \cdot 2^{-n-1}$,

$\mathrm{IH}(3) \quad p_{n}$ is $\left(E_{n}, k_{n}\right)$ determined.

For each $n$, an integer $J_{n}$ and a sequence $\mathcal{C}_{n}=\left\{c_{j}^{n} \mid j \leq J_{n}\right\}$ such that $c_{0}^{n}=0<c_{1}^{n}<c_{2}^{n}<\ldots<c_{J_{n}}^{n}=1$ will be chosen so that

$\operatorname{IH}(4) \quad d_{n} \in \mathcal{C}_{n}$ and $\mathcal{C}_{i} \subseteq \mathcal{C}_{n}$ if $i \in n$,

$\operatorname{IH}(5) \quad$ if $i \in n$ and $c \in \mathcal{C}_{i}$ then $f_{n}(c)=f_{i}(c)$. 
For each $n$ and each $j \leq J_{n}$ a continuous function $\Phi_{n, j}: p_{n}^{*} \rightarrow \mathbb{R}$ will be found so that there is a name $z_{n, j}$ such that

$\operatorname{IH}(6) \quad p_{n} \Vdash_{\mathbb{S}_{\omega_{2}}} " H\left(z_{n, j}\right)=\Phi_{n, j}(G)$ " for each $j \in J_{n}$.

A function $Z_{n, j}: 2^{\omega_{2} \times \omega} \rightarrow \mathbb{R}$ will also be constructed so that

$\operatorname{IH}(7) \quad p_{n} \Vdash_{\mathbb{S}_{\omega_{2}}} " z_{n, j}=Z_{n, j}(G) "$,

$\operatorname{IH}(8) \quad$ if $Z_{n, j}(x)=Z_{n, j}(y)$ then $\Pi_{\omega_{2}, 1}(x)=\Pi_{\omega_{2}, 1}(y)$,

and, with $C_{n, m, j}$ denoting the image of $p_{n}$ under the mapping $Z_{m, j}$,

$\mathrm{IH}(9) \quad$ if $m<k \leq n, j \in J_{m}$ and $i \in J_{k}$ then $C_{n, m, j} \cap C_{n, k, i}=\emptyset$.

By $\left[A_{m, j}, B_{m, j}\right]$ will be denoted the interval with endpoints $\left(f_{m}+H\right)\left(c_{j}^{m}\right)$ and $\left(f_{m}+H\right)\left(c_{j+1}^{m}\right)$. Observe that $\mathrm{IH}(5)$ implies that the definition of $\left[A_{m, j}, B_{m, j}\right]$ does not change at later stages of the induction.

$\mathrm{IH}(10) \quad$ the image of $p_{n}$ under $f_{n} \circ Z_{n, j}+\Phi_{n, j} \circ \Pi_{\omega_{2}, 1}$ contains the interval $\left[A_{n, j}, B_{n, j}\right]$ for each $j \in J_{n}$.

For $x \in[0,1]$ let $p_{n, m, j}^{x}$ be the join of all conditions $p_{n} \mid \sigma$ such that $\sigma$ : $E_{n} \times k_{n} \rightarrow 2$ is consistent with $p_{n}$ and $x$ belongs to the image of $\left(p_{n} \mid \sigma\right)^{*}$ under the mapping $f_{n} \circ Z_{m, j}+\Phi_{m, j} \circ \Pi_{\omega_{2}, 1}$. The following is the key inductive requirement.

$\mathrm{IH}(11) \quad$ if $x \in\left[A_{m, j}, B_{m, j}\right]$ then $\left(p_{n+1, m, j}^{x}, k_{n+1}\right)<_{E_{n+1}}\left(p_{n, m, j}^{x}, k_{n}\right)$.

Assuming that the induction can be completed, let $f=\lim _{n \rightarrow \infty} f_{n}$. It will be shown that there is a condition $p_{\omega} \in \mathbb{S}_{\omega_{2}}$ which forces that $T(f)$ belongs to the image of $N(f)$ under $f$. This contradiction will establish the theorem because $\mathrm{IH}(1)$ and $\mathrm{IH}(2)$ obviously guarantee that $f \in X$.

Let $m$ be an integer such that there is some $j \in J_{m}$ such that $\left[c_{j}^{m}, c_{j+1}^{m}\right] \subseteq$ $N(f)$ and $T(f) \in\left[A_{m, j}, B_{m, j}\right]$. The integers $m$ and $j$ must exist because the endpoints of $N(f)$ belong to $D$ and so $N(f)=\left[c_{i}^{m}, c_{k}^{m}\right]$ for some $m, i$ and $k$. Furthermore, from $\mathrm{IH}(5)$ it follows that

$$
\left[H+f\left(c_{i}^{m}\right), H+f\left(c_{k}^{m}\right)\right]=\left[H+f_{m}\left(c_{i}^{m}\right), H+f_{m}\left(c_{k}^{m}\right)\right]=\bigcup_{i \leq v<k}\left[A_{m, v}, B_{m, v}\right] .
$$

There must, therefore, be some $j$ between $i$ and $k-1$ which is suitable.

It follows from $\mathrm{IH}(10)$ that the range of $f_{m} \circ Z_{m, j}+\Phi_{m, j} \circ \Pi_{\omega_{2}, 1}$ contains $T(f)$ and so $p_{m, m, j}^{T(f)} \neq \emptyset$. From $\mathrm{IH}(11)$ it follows that

$$
\left(p_{n+1, m, j}^{T(f)}, k_{n+1}\right)<_{E_{n+1}}\left(p_{n, m, j}^{T(f)}, k_{n}\right)
$$

for each $n \geq m$ and so there is a condition $p_{\omega} \in \mathbb{S}_{\omega_{2}}$ such that $p_{\omega} \leq$ $p_{n, m, j}^{T(f)}$ for $n \geq m$. It follows that $T(f)$ belongs to the image of $p_{\omega}^{*}$ under $f \circ Z_{m, j}+\Phi_{m, j} \circ \Pi_{\omega_{2}, 1}$. Furthermore, because the diameters of the images 
of $\left(p_{n, m, j}^{T(f)}\right)^{*}$ under $f_{n} \circ Z_{m, j}+\Phi_{m, j}$ approach 0 as $n$ increases, it follows that $f \circ Z_{m, j}+\Phi_{m, j} \circ \Pi_{\omega_{2}, 1}$ has constant value $T(f)$ on $p_{\omega}^{*}$.

It follows that $p_{\omega} \Vdash$ " $f \circ Z_{m, j}+\Phi_{m, j} \circ \Pi_{\omega_{2}, 1}(G)=T(f)$ ". From $\operatorname{IH}(6)$ and the fact that $p_{\omega} \leq p_{m}$ it follows that $p_{\omega} \Vdash " H\left(z_{m, j}\right)=\Phi_{m, j} \circ \Pi_{\omega_{2}, 1}(G) "$ and from $\operatorname{IH}(7)$ that $p_{\omega} \Vdash " z_{m, j}=Z_{m, j}(G)$ ". Therefore $p_{\omega} \Vdash " f\left(z_{m, j}\right)+$ $H\left(z_{m, j}\right)=T(f)$ " and this is a contradiction because $z_{m, j} \in\left[c_{j}^{m}, c_{j+1}^{m}\right] \subseteq$ $N(f)$ by definition.

To carry out the induction suppose that $f_{n},\left\{\Phi_{n, j} \mid j \in J_{n}\right\}$ and $\left\{Z_{n, j} \mid\right.$ $\left.j \in J_{n}\right\}$ as well as conditions $p_{n} \in \mathbb{S}_{\omega_{2}}$ have all been defined for $n \leq K$. To begin, let $0=c_{0}^{K+1}<c_{1}^{K+1}<\ldots<c_{J_{K+1}}^{K+1}=1$ be such that

- $\left\{d_{K}\right\} \cup \mathcal{C}_{K} \subseteq \mathcal{C}_{K+1}=\left\{c_{i}^{K+1} \mid i \leq J_{K+1}\right\}$,

- the diameter of the $f_{K}$-image of $\left[c_{i}^{K+1}, c_{i+1}^{K+1}\right]$ is less than $\varepsilon_{K} \cdot 2^{-K-4}$, - $0<\left|H\left(c_{i}^{K+1}\right)-H\left(c_{i+1}^{K+1}\right)\right|<\varepsilon_{K} \cdot 2^{-K-3}$.

The first condition ensures that $\mathrm{IH}(4)$ is satisfied. The second is easily arranged using uniform continuity. The last condition can be satisfied by a further refinement using the Darboux property of $H$.

Note that $\Pi_{\omega_{2}, 1}\left(p_{K}^{*}\right)$, the image of $p_{K}^{*}$ under $\Pi_{\omega_{2}, 1}$, is perfect and so, for each $i \in J_{K+1}$ it is possible to find $\Phi_{K+1, i}: \Pi_{\omega_{2}, 1}\left(p_{K}^{*}\right) \rightarrow \mathbb{R}$ such that

- $\Phi_{K+1, i}$ is a continuous mapping,

- the image of $p_{K}^{*}$ under $\Phi_{K+1, i} \circ \Pi_{\omega_{2}, 1}$ is the interval with endpoints $H\left(c_{i}^{K+1}\right)$ and $H\left(c_{i+1}^{K+1}\right)$,

- if $(m, j) \neq(K+1, i)$ then $\Phi_{K+1, i} \circ \Pi_{\omega_{2}, 1}(x) \neq \Phi_{m, j} \circ \Pi_{\omega_{2}, 1}(x)$ for every $x \in p_{K}^{*}$,

- $\Phi_{K+1, i}$ is finite-to-one.

Observe that the last point implies that $\Phi_{K+1, i} \circ \Pi_{\omega_{2}, 1}(G)$ does not belong to the ground model $V$.

In any generic extension there must be a real between $c_{i}^{K+1}$ and $c_{i+1}^{K+1}$ at which $H$ takes on the value $\Phi_{K+1, i} \circ \Pi_{\omega_{2}, 1}(G)$ because $H$ is assumed to be Darboux. Let $z_{K+1, i}$ be a name for such a real. It follows from the choice of $\Phi_{K+1, i}$ that $1 \Vdash$ " $z_{m, j} \neq z_{K+1, i}$ " for each $m \leq K+1$ and $j \in J_{m}$ such that $(K+1, i) \neq(m, j)$.

Now find $k$ and $p$ such that

- $\left(p, k_{K}\right)<_{E_{K+1}}\left(p_{K}, k_{K}\right)$,

- $p$ is $\left(E_{K+1}, k\right)$ determined,

- for each $\sigma: E_{K+1} \times k_{K} \rightarrow 2$ which is consistent with $p_{K}$ and for each $m \leq K, j \in J_{m}$ and for each $x$ in the image of $\left(p_{K} \mid \sigma\right)^{*}$ under $f_{K} \circ Z_{m, j}+$ $\Phi_{m, j} \circ \Pi_{\omega_{2}, 1}$ there is some $\sigma^{\prime}: E_{K+1} \times k \rightarrow 2$ such that

- $\sigma^{\prime}$ is consistent with $p$,

$-\sigma \subseteq \sigma^{\prime}$, 
- the distance from $x$ to the image of $\left(p \mid \sigma^{\prime}\right)^{*}$ under $f_{K} \circ Z_{m, j}+\Phi_{m, j} \circ$ $\Pi_{\omega_{2}, 1}$ is less than $\varepsilon_{K} \cdot 2^{-K-4}$,

- the diameter of the image of $(p \mid \sigma)^{*}$ under $f_{K} \circ Z_{m, j}+\Phi_{m, j} \circ \Pi_{\omega_{2}, 1}$ is less than $\varepsilon_{K} \cdot 2^{-K-4}$ for each $\sigma: E_{K+1} \times k \rightarrow 2$ which is consistent with $p$.

Now let $k_{K+1}$ and $\bar{p}$ be such that

- $\left(\bar{p}, k_{K+1}\right)<_{E_{K+1}}(p, k)$,

- $\bar{p}$ is $\left(E_{K+1}, k_{K+1}\right)$ determined.

Because $V$ is closed under $H$ and $\Phi_{m, j} \circ \Pi_{\omega_{2}, 1}(G) \notin V$ it follows that $z_{K+1, i}$ is a name for a real which does not belong to $V$. Lemma 3.1 can therefore be used $J_{K+1}$ times to find a condition $q$ such that $\left(q, k_{K+1}\right) \leq_{E_{K+1}}$ $\left(\bar{p}, k_{K+1}\right)$ and for each $i \in J_{K+1}$ there is a function $Z_{K+1, i}: q^{*} \stackrel{\rightarrow}{\rightarrow}$ $\left[c_{i}^{K+1}, c_{i+1}^{K+1}\right]$ such that

- $q \Vdash$ " $z_{K+1, i}=Z_{K+1, i}(G)$ ",

- $Z_{K+1, i}(x) \neq Z_{K+1, i}(y)$ unless $\Pi_{\omega_{2}, 1}(x)=\Pi_{\omega_{2}, 1}(y)$.

Now observe that if $(m, j) \neq(K+1, i)$ then there cannot be $\widehat{q} \leq q$ such that $Z_{m, j}\left\lceil\widehat{q}^{*}=Z_{K+1, i}\left\lceil\widehat{q}^{*}\right.\right.$ because it has already been remarked that $1 \Vdash$ " $z_{m, j} \neq z_{K+1, i}$ " for each $m \leq K+1$ and $j \in J_{m}$ such that $(K+1, i) \neq(m, j)$. It is therefore possible to use Lemma 3.2 repeatedly to find a single condition $p_{K+1}$ such that $\left(p_{K+1}, k_{K+1}\right)<_{E_{K+1}}\left(q, k_{K+1}\right)$ and the image of $p_{K+1}^{*}$ under $Z_{K+1, i}$ is disjoint from the image of $p_{K+1}^{*}$ under $Z_{m, j}$ if $(m, j) \neq(K+1, i)$. Observe that $p_{K+1}$ is $\left(E_{K+1}, k_{K+1}\right)$ determined because $\bar{p}$ is. Hence $\operatorname{IH}(3)$ is satisfied. Now define $C_{K+1, K+1, i}$ to be the range of $Z_{K+1, i}$. This, along with the induction hypothesis, will guarantee that $\mathrm{IH}(6)-\mathrm{IH}(9)$ are all satisfied.

For integers $m \leq K+1, j \in J_{m}$ let $\left\{\left[u_{m, j, v}^{0}, u_{m, j, v}^{1}\right] \mid v \in L_{m, j}\right\}$ be a partition of $\left[A_{m, j}, B_{m, j}\right]$ into intervals of length $\varepsilon_{K} \cdot 2^{-K-2}$. Now, for each $\sigma: E_{K+1} \times k_{K+1} \rightarrow 2$ and for each pair of integers $m \leq K+1, j \in J_{m}$ and for each $v \in L_{m, j}$ let $W[\sigma, m, j, v]$ be a perfect, nowhere dense subset of

$$
\left(f_{K}+\Phi_{m, j} \circ\left(\Pi_{\omega_{2}, 1}\left\lceil\left(p_{K+1} \mid \sigma\right)\right) \circ Z_{m, j}^{-1}\right)^{-1}\left[u_{m, j, v}^{0}-\frac{\varepsilon_{K}}{2^{K+3}}, u_{m, j, v}^{1}+\frac{\varepsilon_{K}}{2^{K+3}}\right]\right.
$$

if this is possible. By choosing smaller sets, if necessary, it may be assumed that the sets $W[\sigma, m, j, v]$ are pairwise disjoint and that $W[\sigma, m, j, v] \cap$ $\mathcal{C}_{K+1}=\emptyset$. Then define $F_{\sigma, m, j, v}: W[\sigma, m, j, v] \rightarrow\left[u_{m, j, v}^{0}, u_{m, j, v}^{1}\right]$ to be any continuous surjection and let $f_{\sigma, m, j, v}=F_{\sigma, m, j, v}-\Phi_{m, j} \circ \Pi_{\omega_{2}, 1} \circ Z_{m, j}^{-1}$. Note that $\mathrm{IH}(8)$ implies that $\Pi_{\omega_{2}, 1} \circ Z_{m, j}^{-1}$ is a function even though $Z_{m, j}$ is not one-to-one.

Similarly, for each $i \leq J_{K+1}$ let $W_{i}$ be a perfect, nowhere dense subset of $\left[c_{i}^{K+1}, c_{i+1}^{K+1}\right]$ disjoint from each $W[\sigma, m, j, v]$ and define $F_{i}: W_{i} \rightarrow$ $\left[A_{K+1, i}, B_{K+1, i}\right]$ to be a continuous surjection. Then let $f^{i}=F_{i}-\Phi_{K+1, i} \circ$ $\Pi_{\omega_{2}, 1} \circ Z_{K+1, i}^{-1}$. Notice that the domains of all the functions $f_{\sigma, m, j, v}$ and $f^{i}$ 
are pairwise disjoint. Hence it is possible to find $f_{K+1}: I \rightarrow \mathbb{R}$ extending each of these functions in such a way that $\varrho\left(f_{K+1}, f_{K}\right)$ does not exceed

$$
\max \left\{\left|f_{K+1}(y)-f_{K}(y)\right|: y \in\left(\bigcup_{i} W_{i}\right) \cup\left(\bigcup_{\sigma, m, j, v} W[\sigma, m, j, v]\right)\right\}
$$

and, moreover, because $W[\sigma, m, j, v] \cap \mathcal{C}_{K+1}=\emptyset$ and $W_{i} \cap \mathcal{C}_{K+1}=\emptyset$, it may also be arranged that $f_{K+1}(c)=f_{K}(c)$ if $c \in \mathcal{C}_{K}$. Therefore $\operatorname{IH}(5)$ is satisfied as well as $\mathrm{IH}(0)$. Observe that $\mathrm{IH}(10)$ is satisfied because the choice of $F_{j}$ ensured that it maps $W_{j}$ onto $\left[A_{K+1, j}, B_{K+1, j}\right]$. Since $f_{K+1}\left\lceil W_{j}=\right.$ $F_{j}-\Phi_{K+1, j} \circ \Pi_{\omega_{2}, 1} \circ Z_{K+1, j}^{-1}$ it follows that $f_{K+1} \circ Z_{K+1, j}+\Phi_{K+1, j} \circ \Pi_{\omega_{2}, 1}$ maps $p_{K+1}$ onto $\left[A_{K+1, j}, B_{K+1, j}\right]$.

To see that $\mathrm{IH}(2)$ holds it suffices to consider only

$$
f_{K+1} \uparrow\left(\bigcup_{i} W_{i}\right) \cup\left(\bigcup_{\sigma, m, j, v} W[\sigma, m, j, v]\right)
$$

because $f_{K+1}$ was defined not to exceed this bound. Consider first $y \in$ $W[\sigma, m, j, v]$. Then $\left|f_{K+1}(y)-f_{K}(y)\right|$ is equal to

$$
\left|f_{\sigma, m, j, v}(y)-f_{K}(y)\right|=\left|F_{\sigma, m, j, v}(y)-\left(\Phi_{m, j} \circ \Pi_{\omega_{2}, 1} \circ Z_{m, j}^{-1}(y)+f_{K}(y)\right)\right| .
$$

Next, the definition of $F_{\sigma, m, j, v}$ implies that $F_{\sigma, m, j, v}(y) \in\left[u_{m, j, v}^{0}, u_{m, j, v}^{1}\right]$. By the definition of $W[\sigma, m, j, v]$,

$$
\left(f_{K}+\Phi_{m, j} \circ \Pi_{\omega_{2}, 1} \circ Z_{m, j}^{-1}\right)(y) \in\left[u_{m, j, v}^{0}-\varepsilon_{K} / 2^{-K-3}, u_{m, j, v}^{1}+\varepsilon_{K} / 2^{-K-3}\right]
$$

because $y \in W[\sigma, m, j, v]$. Consequently, $\left|f_{K+1}(y)-f_{K}(y)\right|$ is no greater than the diameter of

$$
\left[u_{m, j, v}^{0}-\varepsilon_{K} / 2^{-K-3}, u_{m, j, v}^{1}+\varepsilon_{K} / 2^{-K-3}\right],
$$

which is $\varepsilon_{K} \cdot 2^{-K-3}+\varepsilon_{K} \cdot 2^{-K-2}+\varepsilon_{K} \cdot 2^{-K-3}=\varepsilon_{K} \cdot 2^{-K-1}$.

On the other hand, if $y \in W_{i}$ then, as before,

$$
\left|f_{K+1}(y)-f_{K}(y)\right|=\left|F_{i}(y)-\left(\Phi_{m, j} \circ \Pi_{\omega_{2}, 1} \circ Z_{m, j}^{-1}(y)+f_{K}(y)\right)\right| .
$$

Recall that $\Phi_{K+1, i}$ is chosen to map onto $\left[H\left(c_{i}^{K+1}\right), H\left(c_{i+1}^{K+1}\right)\right]$; moreover, because $y \in\left[c_{i}^{K+1}, c_{i+1}^{K+1}\right]$ it follows from the choice of $\mathcal{C}_{K+1}$ that

$$
f_{K}(y) \in\left[f_{K}\left(c_{i}^{K+1}\right)-\varepsilon_{K} / 2^{-k-4}, f_{K}\left(c_{i}^{K+1}\right)+\varepsilon_{K} / 2^{-k-4}\right]
$$

and so $\Phi_{m, j} \circ \Pi_{\omega_{2}, 1} \circ Z_{m, j}^{-1}(y)+f_{K}(y)$ belongs to $\left[A_{K+1, i}-\varepsilon_{K} / 2^{-K-4}, B_{K+1, i}\right.$ $\left.+\varepsilon_{K} / 2^{-K-4}\right]$. Furthermore, $F_{i}(y)$ belongs to $\left[A_{K+1, i}, B_{K+1, i}\right]$ by design. By the choice of $\mathcal{C}_{K+1}$ the diameter of $\left[A_{K+1, i}, B_{K+1, i}\right]$ is less than $\varepsilon_{K} / 2^{-K-4}+$ $\varepsilon_{K} / 2^{-K-3}$ and so the diameter of

$$
\left[A_{K+1, i}-\varepsilon_{K} / 2^{-K-4}, B_{K+1, i}+\varepsilon_{K} / 2^{-K-4}\right]
$$

is no greater than $\varepsilon_{K} / 2^{-K-1}$ and it follows that $\left|f_{K+1}(y)-f_{K}(y)\right|<$ $\varepsilon_{K} \cdot 2^{-K-1}$. 
Now all of the induction hypotheses have been shown to be satisfied except for $\mathrm{IH}(1)$ and $\mathrm{IH}(11)$. To verify $\mathrm{IH}(11)$ suppose that $m \leq K, j \in J_{m}$ and $x \in\left[A_{m, j}, B_{m, j}\right]$. It follows that there is some $v \in L_{m, j}$ such that $x \in\left[u_{m, j, v}^{0}, u_{m, j, v}^{1}\right]$. Suppose also that $\sigma: E_{K+1} \times k_{K} \rightarrow 2$ is consistent with $p_{K, m, j}^{x}$. It follows that there is some $\sigma^{\prime}: E_{K+1} \times k \rightarrow 2$ such that

- $\sigma^{\prime}$ is consistent with $p$,

- $\sigma \subseteq \sigma^{\prime}$,

- the distance from $x$ to the image of $\left(p \mid \sigma^{\prime}\right)^{*}$ under $f_{K} \circ Z_{m, j}+\Phi_{m, j} \circ \Pi_{\omega_{2}, 1}$ is less than $\varepsilon_{K} \cdot 2^{-K-4}$.

It suffices to show that $\tau$ is consistent with $p_{K+1, m, j}^{x}$ for each $\tau: E_{K+1} \times$ $k_{K+1} \rightarrow 2$ such that $\sigma^{\prime} \subseteq \tau$; the reason for this is that $k_{K+1}$ was chosen so that $\left(\bar{p}, k_{K+1}\right) \leq_{E_{K+1}}\left(p_{K}, k\right)$ and $\left(p_{K+1}, k_{K+1}\right) \leq_{E_{K+1}}\left(\bar{p}, k_{K+1}\right)$. Recall that the diameter of the image of $\left(p \mid \sigma^{\prime}\right)^{*}$ under $f_{K} \circ Z_{m, j}+\Phi_{m, j} \circ \Pi_{\omega_{2}, 1}$ is less than $\varepsilon_{K} \cdot 2^{-K-4}$ because $\sigma^{\prime}: E_{K+1} \times k \rightarrow 2$ is consistent with $p$. Because the distance from $x$ to the image of $\left(p \mid \sigma^{\prime}\right)^{*}$ under $f_{K} \circ Z_{m, j}+$ $\Phi_{m, j} \circ \Pi_{\omega_{2}, 1}$ is less than $\varepsilon_{K} \cdot 2^{-K-4}$ it must be that this image is contained in $\left[u_{m, j, v}^{0}-\varepsilon_{K} \cdot 2^{-K-3}, u_{m, j, v}^{1}-\varepsilon_{K} \cdot 2^{-K-3}\right]$. Because $p_{K+1}<p$ it follows that the image of $\left(p_{K+1} \mid \tau\right)^{*}$ under $f_{K} \circ Z_{m, j}+\Phi_{m, j} \circ \Pi_{\omega_{2}, 1}$ is contained in $\left[u_{m, j, v}^{0}-\varepsilon_{K} \cdot 2^{-K-3}, u_{m, j, v}^{1}-\varepsilon_{K} \cdot 2^{-K-3}\right]$ and so $W[\tau, m, j, v] \neq \emptyset$. The choice of $F_{\tau, m, j, v}$ ensures that it maps $W[\tau, m, j, v]$ onto $\left[u_{m, j, v}^{0}, u_{m, j, v}^{1}\right]$ and therefore $f_{K+1}+\Phi_{m, j} \circ\left(\Pi_{\omega_{2}, 1} \uparrow\left(p_{K+1} \mid \tau\right)\right) \circ Z_{m, j}^{-1}$ maps $W[\tau, m, j, v]$ onto $\left[u_{m, j, v}^{0}, u_{m, j, v}^{1}\right]$. Hence $\tau$ is consistent with $p_{K+1, m, j}^{x}$.

Finally, choose $\varepsilon_{K+1}$ so that the neighbourhood of $f_{K+1}$ of radius $\varepsilon_{K+1}$ is contained in $U_{K+1}$.

COROLlaRY 4.1. If set theory is consistent then it is consistent that for every Darboux function $F$ there is a nowhere constant continuous function $f$ such that $F+f$ is also Darboux.

Pr o of. The model to use is the one for Theorem 4.1. Given a Darboux $F$ to obtain a nowhere constant continuous $f$ use the fact that the set of nowhere constant functions is comeagre in $\mathcal{C}(I, \mathbb{R})$.

5. Further remarks. It should be observed that the function $f$ in Corollary 4.1 has very few nice properties other than continuity. It is natural to ask the following question.

Question 5.1. Is there a Darboux function $H: I \rightarrow \mathbb{R}$ such that $H+f$ is not Darboux for every non-constant, differentiable function $f$ ?

The answer to Question 5.1 for functions with continuous derivative is positive. The same question can be asked with absolutely continuous in place 
of differentiable. Recall that differentiable functions satisfy the property $T_{1}$ of Banach [9].

Definition 5.1. A function $F: \mathbb{R} \rightarrow \mathbb{R}$ satisfies $T_{1}$ if and only if the set of all $x$ such that $f^{-1}\{x\}$ is infinite has measure zero.

Banach showed that differentiable functions satisfy $T_{1}$. Question 5.1 is of interest for differentiable functions because Corollary 2.1 shows that a strengthening of $T_{1}$ yields a positive theorem.

Another potentially interesting direction to pursue would be to ask whether the size of the set of continuous functions in Theorem 4.1 can be increased.

Question 5.2. Is there a Darboux function F such that the set of continuous functions $f$ with $F+f$ Darboux is not comeagre?

Question 5.3. Is there a Darboux function F such that the set of continuous functions $f$ with $F+f$ Darboux does not have measure one?

In [5] it is shown that the answers to these questions are consistently negative.

In the same paper the authors also consider not only sums of a Darboux function and a continuous function, but also products and other algebraic constructions. It is not difficult to check that everything that has been established in this paper for sums also holds for products, but it is not clear that this must always be so.

Question 5.4. If there is a Darboux function $F$ such that $F+g$ is not Darboux for every nowhere constant function $g$, must it also be the case that there is a Darboux function $F$ such that $F \cdot g$ is not Darboux for every nowhere constant function $g$ ? What about the opposite implication?

\section{References}

[1] J. E. Baumgartner and R. Laver, Iterated perfect set forcing, Ann. Math. Logic 17 (1979), 271-288.

[2] A. Bruckner, Differentiation of Real Functions, Lecture Notes in Math. 659, Springer, Berlin, 1978.

[3] A. Bruckner and J. Ceder, Darboux continuity, Jahresber. Deutsch. Math.-Verein. 67 (1965), 100.

[4] - - - On the sums of Darboux functions, Proc. Amer. Math. Soc. 51 (1975), 97-102.

[5] B. Kirchheim and T. Natkaniec, On universally bad Darboux functions, Real Anal. Exchange 16 (1990-91), 481-486.

[6] P. Komjáth, A note on Darboux functions, ibid. 18 (1992-93), 249-252.

[7] A. Miller, Mapping a set of reals onto the reals, J. Symbolic Logic 48 (1983), 575584 . 
[8] T. Radakovič, Über Darbouxsche und stetige Funktionen, Monatsh. Math. Phys. 38 (1931), 117-122.

[9] S. Saks, Theory of the Integral, Hafner, New York, 1937.

DEPARTMENT OF MATHEMATICS

YORK UNIVERSITY

TORONTO, ONTARIO

CANADA M3J 1P3

E-mail: STEPRANS@NEXUS.YORKU.CA

Received 23 March 1993;

in revised form 24 February 1994 